\title{
Hipovitaminosis D y antiepilépticos: revisión de la evidencia y recomendaciones
}

\author{
Agustina De Santis*, Valentina Catenaccio†, Noelia Speranza
}

\section{Resumen}

La vitamina $\mathrm{D}$ regula funciones óseas y extra-óseas. La hipovitaminosis $\mathrm{D}$ está re emergiendo como problema de salud. Diversos factores pueden determinarla (exposición solar, malabsorción, obesidad, sedentarismo y exposición a antiepilépticos). El objetivo de este trabajo fue revisar la evidencia sobre la asociación entre la hipovitaminosis $D$ y el tratamiento con antiepilépticos.

Métodos: se realizó una revisión descriptiva de la evidencia disponible en los últimos 10 años en las bases de datos Pubmed y Scielo.

Resultados: se analizaron 5 estudios que cumplían con los criterios de búsqueda preespecificados. Cuatro estudios eran prospectivos, 2 realizados en India (uno en niños expuestos a carbamazepina y otro en adultos con difenilhidantoína o ácido valproico), otro en Grecia en niños con carbamazepina o ácido valproico y en los 3 se evidenció el déficit; el cuarto estudio fue realizado en Alemania en niños expuestos a varios antiepilépticos y no se halló asociación. Un estudio fue retrospectivo, realizado en Estados Unidos en adultos expuestos a varios antiepilépticos y se halló asociación.

Discusión: se destaca el escaso número de estudios hallados, son observacionales, de bajo número de pacientes y corta duración. Se evidenció que la hipovitaminosis $D$ fue un hecho frecuente si bien por el tipo de estudios no puede establecerse una relación causal. Aún no es clara la magnitud del problema pues no existe consenso sobre qué niveles de vitamina $\mathrm{D}$ se consideran deficientes. La profilaxis con vitamina $\mathrm{D}$ en pacientes con antiepilépticos parece racional a pesar de las limitantes de la información disponible. Es necesario continuar caracterizando la asociación y sus implicancias clínicas.

Palabras clave: Deficiencia de vitamina D

Anticonvulsivantes.

Key words: $\quad$ Vitamin D deficiency

Anticonvulsants.

\footnotetext{
* Bachiller. Ayudante Dpto. de Farmacología.

† Pediatra. Asistente Dpto. de Farmacología.

‡ Pediatra. Prof. Agregada del Dpto. de Farmacología.

Los autores declaran no conflicto de intereses.

Recibido 9/4/19

Aprobado 17/6/19
} 


\section{Introducción}

La vitamina $\mathrm{D}$ es una vitamina liposoluble, considerada también una hormona que regula la homeostasis mineral y mantiene la concentración de calcio dentro de los límites normales. Protege la integridad del esqueleto, modula el crecimiento y la diferenciación celular. Existe una amplia distribución de sus receptores en todo el organismo, en especial a nivel del hueso, aparato digestivo y riñón. Además de su rol en la homeostasis del calcio, la vitamina $\mathrm{D}$ regula otras funciones denominadas extraóseas, con posibles beneficios, como la regulación del sistema inmune, la función muscular, el sistema cardiovascular y metabólico $^{(1-3)}$.

Existe cada vez mayor evidencia acerca del déficit de vitamina $\mathrm{D}$ en la población ${ }^{(3,4)}$. Una revisión realizada en 2009 por la International Osteoporosis Foundation and National Osteoporosis Foundation tenía por objetivo conocer el estatus de la vitamina $\mathrm{D}$ en diferentes regiones del mundo e identificar sus determinantes. Esta reportó que la hipovitaminosis D se encuentra ampliamente extendida en los seis continentes y está reemergiendo como problema de salud pública global $^{(3)}$.

La prevalencia de hipovitaminosis D (considerada como menor a $30 \mathrm{ng} / \mathrm{ml}$ ) en Estados Unidos es de 76\%, según datos del centro nacional de salud y nutrición NANHES (National Health and Nutrition Examination Survey) $)^{(5)}$.

A nivel regional, en Argentina se han publicado varios trabajos acerca de la prevalencia del déficit de vitamina D en ese país. En un estudio publicado en 2015 el promedio ponderado de prevalencia del déficit de 25 hidroxi-vitamina D (25 OHD) fue de 43,3\% (436/1.007 personas) considerando como punto de corte un valor menor a $40 \mathrm{ng} / \mathrm{ml}^{(6)}$.

En Uruguay se han estudiado los niveles de vitamina D en poblaciones especiales como embarazadas, mujeres posmenopáusicas y pacientes en hemodiálisis crónica. Considerando hipovitaminosis $\mathrm{D}$ a niveles menores de $30 \mathrm{ng} / \mathrm{ml}$, en embarazadas se observó una frecuencia de $96 \%$ y en pacientes en hemodiálisis crónica de $97 \%{ }^{(7-9)}$.

Diversos factores pueden influir en los niveles de vitamina D, entre ellos la baja exposición a la luz solar, la presencia de enfermedades malabsortivas, la obesidad, el sedentarismo y también la exposición a fármacos que interfieren en el metabolismo de ésta, por ejemplo los fármacos antiepilépticos $(\mathrm{AE})^{(4)}$.

Del punto de vista teórico es conocida la interacción de los AE y el descenso de los niveles séricos de vitamina $\mathrm{D}$, principalmente aquellos inductores de las enzimas hepáticas del citocromo P450 (CYP450) como fenobarbital (FB), difenilhidantoína (DFH), carbamazepina
(CBZ) y primidona. Dichos AE estimulan el metabolismo de la vitamina $\mathrm{D}$ con el consecuente descenso de sus niveles plasmáticos. También existe interacción para aquellos AE no inductores de las enzimas hepáticas, incluso inhibidores de éstas, como el ácido valproico (VPA). Este fármaco produce inhibición de la enzima 25 hidroxilasa (reacción enzimática de bioactivación de la vitamina D). Otros mecanismos propuestos son la disminución de la absorción de calcio, efectos directos sobre las células óseas, deficiencia de calcitonina y resistencia a la hormona paratiroidea ${ }^{(10)}$.

A pesar de este conocimiento, la evidencia científica sobre esta asociación y sus riesgos es escasa y del punto de vista clínico no se ha extendido el uso profiláctico de vitamina $\mathrm{D}$ en pacientes que utilizan $\mathrm{AE}$, pese a la existencia de recomendaciones actuales ${ }^{(11-13)}$.

El objetivo de este trabajo fue revisar la evidencia acerca de la asociación entre la hipovitaminosis D y el tratamiento con AE.

\section{Metodología}

Se realizó una revisión descriptiva sobre el tema de la evidencia disponible en los últimos diez años a través de las bases de datos Pubmed y SciElo.

Para la búsqueda bibliográfica en Pubmed se utilizaron las palabras clave Anticonvulsants and Vitamin D y fue limitada a: Clinical Trial, Clinical Study, Observational Study, Multicenter Study, Controlled Clinical Trial, Meta-Analysis, Randomized Controlled Trial, en humanos, con fecha de publicación en los últimos diez años. Se analizaron artículos en idioma español e inglés.

Con los mismos filtros se realizó una segunda búsqueda con las palabras clave Anticonvulsants and Vitamin D Deficiency.

La búsqueda en SciElo incluyó las palabras claves Vitamina d y Antiepilépticos utilizando como límite Regional, sin límite temporal.

Del análisis de los títulos o resúmenes se excluyeron aquellos estudios que no hicieran referencia a los niveles de vitamina $\mathrm{D}$, o que incluyeran pacientes con patologías que coadyuvan al déficit de vitamina D (parálisis cerebral, neurofibromatosis, enfermedades disabsortivas, insuficiencia renal, hepática, entre otras).

Las autoras analizaron todos los estudios encontrados a texto completo que se ajustaban al objetivo del trabajo.

La búsqueda y análisis fue realizada en el mes de febrero 2019.

\section{Resultados}

En la base de datos SciElo no se encontraron estudios que abordaran el tema de interés. 
Tabla 1. Características de los estudios que abordan hipovitaminosis D.

\begin{tabular}{|c|c|c|c|c|c|c|c|c|c|}
\hline Ref. & f. Año & $\begin{array}{l}\text { Tipo de } \\
\text { estudio }\end{array}$ & País & $\begin{array}{l}\text { Duración } \\
\text { máxima } \\
\text { (meses) }\end{array}$ & $\begin{array}{l}N^{\circ} \text { de } \\
\text { ptes. }\end{array}$ & Tipo de ptes. & $\begin{array}{l}\text { Variables de estudio/ } \\
\qquad A E \text { implicado }\end{array}$ & $\begin{array}{c}\text { Punto corte } \\
\text { deficit }\end{array}$ & Resultados \\
\hline 14 & 2010 & $\begin{array}{l}\text { Observacional } \\
\text {, prospectivo }\end{array}$ & India & 3 & 66 & Adultos epilépticos & $\begin{array}{l}\text { Metabolismo } \\
\text { fosfocálcico; DFH y } \\
\text { VPA. Respuesta con } \\
\text { suplementación de } \\
\text { calcio y vit. D; DFH y } \\
\text { VPA }\end{array}$ & SD & $\begin{array}{l}\text { Disminución vit. D: de } \\
25,19 \text { a 19,76 } \mathrm{ng} / \mathrm{ml} \\
\text { ( } p<0,001 \text { ) Con } \\
\text { suplementación: } \\
\text { aumento significativo } \\
\text { de vit. D: de } 24,36 \text { a } \\
31,53 \mathrm{ng} / \mathrm{ml} \\
(p<0,0005) .\end{array}$ \\
\hline 15 & 2010 & $\begin{array}{l}\text { Observacional } \\
\text {, prospectivo }\end{array}$ & India & 6 & 32 & Niños epilépticos & $\begin{array}{l}\text { Cambios en los } \\
\text { niveles de vit. D y } \\
\text { metabolismo } \\
\text { fosfocálcico; DFH y } \\
\text { VPA }\end{array}$ & $<20 \mathrm{ng} / \mathrm{ml}$ & $\begin{array}{l}\text { Disminución de vit. D: } \\
\text { de } 14,45 \mathrm{ng} / \mathrm{ml} \mathrm{a} \\
11,31 \mathrm{ng} / \mathrm{ml} \\
(\mathrm{p}=0,023) ; 28 \% \\
\text { hipovitaminosis D }\end{array}$ \\
\hline 17 & 2012 & $\begin{array}{l}\text { Estudio } \\
\text { transversal }\end{array}$ & Alemania & 144 & 128 & Niños epilépticos & $\begin{array}{l}\text { Prevalencia de } \\
\text { alteraciones en el } \\
\text { metabolismo } \\
\text { fosfocálcico; CBZ, } \\
\text { VPA, oxcarbazepina, } \\
\text { topiramato y } \\
\text { levetiracetam }\end{array}$ & $<10 \mathrm{ng} / \mathrm{ml}$ & $\begin{array}{l}13,3 \% \text { de } \\
\text { hipovitaminosis D }\end{array}$ \\
\hline 18 & 2005 & $\begin{array}{l}\text { Observacional } \\
\text {, prospectivo }\end{array}$ & Grecia & 144 & 51 & Niños epilépticos & $\begin{array}{l}\text { Prevalencia de } \\
\text { alteraciones en el } \\
\text { metabolismo de la vit. } \\
\text { D; CBZ y VPA }\end{array}$ & $<10 \mathrm{ng} / \mathrm{ml}$ & $\begin{array}{l}49 \% \text { de } \\
\text { hipovitaminosis D }\end{array}$ \\
\hline 16 & 2014 & $\begin{array}{l}\text { Observacional } \\
\text {, retrospectivo }\end{array}$ & Estados Unidos & 120 & 596 & Adultos epilépticos & $\begin{array}{l}\text { Prevalencia de la } \\
\text { hipovitaminosis D; } \\
\text { CBZ, DFH, FB, } \\
\text { primidona, } \\
\text { oxcarbazepina, } \\
\text { topiramato, } \\
\text { gabapentina, } \\
\text { levetiracetam y } \\
\text { lamotrigina }\end{array}$ & $<20 \mathrm{ng} / \mathrm{ml}$ & $\begin{array}{l}45 \% \text { de } \\
\text { hipovitaminosis } \mathrm{D} \text {. } \\
\text { Mayor } \% \text { con } \mathrm{AE} \\
\text { inductores }(\mathrm{p}<0,02)\end{array}$ \\
\hline
\end{tabular}

Ref.: referencia; Pte.: paciente; AE: antiepiléptico; vit. D: vitamina D; CBZ: carbamazepina, VPA: ácido valproico; SD: sin dato.

En la primera búsqueda en Pubmed se encontraron seis artículos, de los cuales se seleccionaron para analizar tres estudios que evaluaban los niveles sanguíneos de $25 \mathrm{OHD}$ en pacientes que recibían $\mathrm{AE}^{(14-16)}$. Se excluyeron tres estudios debido a que no tenían como variable de análisis la hipovitaminosis. En la segunda búsqueda se hallaron tres estudios, de los cuales se analizó uno ${ }^{(17)}$, porque los otros dos ya se habían analizado ${ }^{(14-16)}$ (tabla $1)$.

El primer estudio analizado era prospectivo, realizado en adultos epilépticos, en India y estudió las concentraciones plasmáticas de $25 \mathrm{OHD}$, fosfatasa alcalina (FA), calcio y fósforo después del inicio del tratamiento con DFH o VPA. Se incluyeron pacientes con diagnósti- co reciente de epilepsia, sin valores previos de vitamina D. En el estudio no se explicita el punto de corte de vitamina D utilizado para definir hipovitaminosis D. Como variable secundaria se estudió el efecto de la suplementación simultánea de calcio y vitamina $\mathrm{D}$ sobre estos parámetros (a las dosis diarias habitualmente recomendadas). Se dividió en dos subgrupos la población estudiada: los pacientes del grupo $\mathrm{A}(\mathrm{n}=34)$ recibían suplementación con calcio elemental $1.000 \mathrm{mg}$ día, de los cuales 19 tomaban DFH y 15 VPA. El grupo $\mathrm{B}(\mathrm{n}=32)$ recibía $1.000 \mathrm{mg}$ de calcio elemental y $400 \mathrm{UI}$ de 25 OHD; 17 de estos pacientes recibían DFH y 15 VPA. Cada 15 días se procedía a evaluación clínica completa y los parámetros bioquímicos fueron medidos a los 30, 60 y 90 días. 
En cuanto a la variable primaria, se observó una disminución significativa de vitamina $\mathrm{D}$ a los 90 días de 25,19 a $19,76 \mathrm{ng} / \mathrm{ml}(\mathrm{p}<0,001)$. En relación con las variables secundarias, en el grupo A postsuplementación a los 60 días, hubo un aumento significativo de la FA (de 78,83 a $88,78 \mathrm{p}<0,001)$ y un descenso significativo en los niveles de calcio sérico $(9,30$ a 9,08 $p<0,05)$. En el grupo B, a los 60 días, se observó un descenso significativo en los niveles de FA (de 81,92 a 66,19 p <0,0005), y un aumento significativo en el calcio sérico (de 9,24 a 9,55 $\mathrm{p}<0,001$ ). A los 90 días en este grupo se documentó un aumento significativo en la vitamina $\mathrm{D}(\mathrm{de} 24,36$ a 31,53 $\mathrm{p}<0,0005)^{(14)}$.

El segundo artículo analizado, también realizado en India, fue prospectivo y evaluó los cambios en los marcadores bioquímicos óseos ( $25 \mathrm{OHD}$, calcio, fósforo, FA y parathormona) en niños entre 2 y 12 años que recibían CBZ. También se midieron los niveles séricos de CBZ para poder establecer una correlación. Se incluyeron 47 niños con epilepsia parcial que iniciaban tratamiento con CBZ en monoterapia, ambulatorios, con dieta normal. De éstos, 32 fueron seguidos por un período de seis meses, se midieron dichos parámetros bioquímicos antes y a los seis meses de iniciado el tratamiento. Se definió hipovitaminosis $\mathrm{D}$ a aquellos valores menores de $10 \mathrm{ng} / \mathrm{ml}$. Se evidenció un decremento significativo en los niveles de $25 \mathrm{OHD}$ a los seis meses de comenzado el tratamiento $(\mathrm{p}=0,023)$. La media de $25 \mathrm{OHD}$ al comienzo fue de $14,45 \mathrm{ng} / \mathrm{ml}$ y luego de seis meses 11,31 $\mathrm{ng} / \mathrm{ml}$ (descenso de 21,7\%). Se observó que de 32 pacientes, $9(28 \%)$ presentaron hipovitaminosis D durante el período de estudio. En relación con los niveles de parathormona (PTH), la media para ésta aumentó significativamente, al inicio fue de $28,6 \mathrm{pg} / \mathrm{ml}$ y a los seis meses fue de 41,9 pg/ml $(\mathrm{p}=0,001)$. La concentración de CBZ en todos los pacientes se encontraba dentro del rango terapéutico y no hubo correlación estadísticamente significativa entre los cambios de los niveles de vitamina $\mathrm{D}$, PTH y otros marcadores óseos con los de $\mathrm{CBZ}^{(15)}$.

El tercer estudio analizado fue observacional retrospectivo y evaluó la prevalencia del déficit de vitamina $D$ en pacientes adultos epilépticos. Se realizó una revisión de historias clínicas de 596 pacientes epilépticos atendidos en una clínica de epilepsia en Atlanta (Estados Unidos). En dicho centro se realiza de manera rutinaria la dosificación de 25 OHD. Se revisaron historias clínicas en un período comprendido entre los años 2008 y 2011. Las variables analizadas fueron niveles de vitamina $D$, fecha de dosificación, AE utilizado en ese momento, edad, sexo y raza. Se categorizaron los niveles de vitamina D en déficit $(<20 \mathrm{ng} / \mathrm{dl})$, borderline (20-29 $\mathrm{ng} / \mathrm{dl})$ y normales (>30 ng/dl), así como también los AE utiliza- dos: AE inductores de la CYP450 (CBZ, DFH, FB y primidona), débilmente inductores (oxcarbazepina y topiramato) y AE no inductores (gabapentina, levetiracetam, lamotrigina). El déficit global de vitamina D fue de $45 \%$. Se encontraron diferencias estadísticamente significativas entre los tres grupos de AE con respecto al déficit de vitamina $\mathrm{D}(\mathrm{p}<0,02)$, observándose $54 \%$ de los tratados con $\mathrm{AE}$ inductores, $50 \%$ con los débilmente inductores y $24 \%$ con los no inductores ${ }^{(16)}$.

Otro estudio analizado fue descriptivo, prospectivo, multicéntrico, de cuatro años de duración, realizado en Alemania. Se consideró deficiencia de vitamina $\mathrm{D}$ a valores menores de $10 \mathrm{ng} / \mathrm{ml}$. Se incluyeron niños de 1 a 18 años con diagnóstico de epilepsia. Los AE utilizados fueron VPA, CBZ, oxcarbazepina, topiramato, lamotrigina y levetiracetam. Se excluyeron aquellos pacientes con enfermedades o medicamentos que interfirieran en el metabolismo fosfocálcico y enfermedades neuromusculares o neurológicas. Las variables estudiadas fueron los niveles plasmáticos de calcio, fósforo, PTH, 25 OHD y FA. Se tomó como grupo control los valores de referencia obtenidos en la última encuesta de salud realizada en Alemania, en 2009. Se incluyeron un total de 128 niños; 76 niñas, 52 varones, media de edad 9 años, todos de raza blanca. Los AE utilizados fueron VPA $(n=67)$, oxcarbazepina $(n=26)$ y lamotrigina $(n=14) . \mathrm{La}$ duración del tratamiento fue de seis meses a tres años con una media de 30 meses. Las concentraciones de calcio fueron menores que el grupo control: se encontró hipocalcemia significativa en $24,4 \%$ de los pacientes $(\mathrm{p}<0,01)$. Con respecto al fosfato, PTH y $25-\mathrm{OHD}$ no hubo diferencias estadísticamente significativas comparados con el control, si bien hubo una tendencia a valores menores de fosfato y $\mathrm{PTH}$ en pacientes tratados con AE. Se encontró hipofosfatemia en $25,4 \%(n=30)$ de los pacientes y deficiencia de vitamina $\mathrm{D}$ en $13,3 \%(\mathrm{n}=17)$. Todos los pacientes presentaron valores de vitamina $\mathrm{D}$ menores a $30 \mathrm{ng} / \mathrm{ml}^{(17)}$.

De las citas del estudio Misra y colaboradores ${ }^{(15)}$, se halló otro trabajo que no se identificó en la búsqueda de Pubmed y que se analizó. Se trata de un estudio observacional, prospectivo, realizado en Grecia, en el que se evaluó los efectos de CBZ y VPA en el metabolismo de la vitamina $\mathrm{D}$ en niños epilépticos sin otras patologías. Se realizó un seguimiento prospectivo de 51 niños eutróficos que consultaban por convulsiones sin tratamiento previo, llevando a cabo una dieta y actividades habituales por tres años. Las variables estudiadas fueron: concentración sérica de $25 \mathrm{OHD}$, PTH, calcio, fósforo, transaminasas y niveles plasmáticos de $\mathrm{AE}$, realizándose mediciones antes de comenzar la terapia y luego cada tres meses. Los criterios de exclusión fueron todas aquellas condiciones clínicas que pudieran coadyu- 
var al déficit de vitamina $\mathrm{D}$ : insuficiencia renal, presencia de enfermedades malabsortivas, esqueléticas o metabólicas, retraso mental. Ninguno de ellos se encontraba bajo tratamiento con vitamina $\mathrm{D}$, calcio, corticoides, diuréticos o alguna otra medicación que interfiera con el metabolismo del calcio durante los tres meses previos. El 49\% de la población estudiada ( 25 de 51 ) presentó hipovitaminosis $\mathrm{D}$, definida como niveles de $25 \mathrm{OHD}$ $<10 \mathrm{ng} / \mathrm{dl}$, y se observó una tendencia decreciente de los niveles de 25 OHD a medida que pasaba el tiempo de uso de AE. Durante el primer año de tratamiento, $37 \%$ (19 de 51) presentó dicho déficit. Se observó una correlación negativa entre los niveles de $25 \mathrm{OHD}$ y PTH. No se encontraron diferencias significativas entre CBZ y VPA en la magnitud del efecto. Se observó una variabilidad estacional en los valores de vitamina $\mathrm{D}$, siendo estos más bajos en invierno que en verano ${ }^{(18)}$.

\section{Desarrollo y discusión}

Se destaca el escaso número de estudios clínicos que abordan el tema tanto a nivel internacional como regional, con un bajo número de pacientes incluidos, tanto en niños como en adultos.

Todos los estudios incluidos fueron observacionales. Los estudios prospectivos incluidos tienen la ventaja de evaluar los valores de vitamina D y metabolismo fosfocálcico al inicio del tratamiento y luego de un tiempo definido de uso, lo cual permite valorar la relación entre el AE utilizado y la duración, observándose que ya existen cambios a los 60 días de tratamiento y en algunos marcadores (FA y calcio sérico) con diferencias estadísticamente significativas ${ }^{(14)}$.

Dado que no se hallaron estudios analíticos, la relación causal con el uso de AE no puede establecerse; por el momento solo se puede describir una tendencia hacia la hipovitaminosis, conforme al sustento teórico ya conocido. Todos estos aspectos dificultan la representatividad e interpretación de los resultados descritos, pero es posible evidenciar que la hipovitaminosis $\mathrm{D}$ es un hecho frecuente con el uso de $\mathrm{AE}$, con valores que llegan casi a $50 \%$ en uno de los estudios analizados ${ }^{(16)}$.

En cuanto a la duración, algunos fueron muy cortos, con un período máximo de observación de tres años, y excepto uno, ninguno evaluó la estacionalidad que es posible observar con este fenómeno ${ }^{(18)}$.

Es importante tener en cuenta que los $\mathrm{AE}$ son frecuentemente utilizados para otras indicaciones como dolor neuropático, cefalea crónica, trastornos del humor, entre otros, y todos los estudios incluidos fueron realizados en pacientes epilépticos, por lo que la prevalencia de la hipovitaminosis D puede estar aún subestimada.
Si bien el problema parece importante en frecuencia, es aún difícil establecer su verdadera magnitud, pues no existe consenso acerca de qué niveles de 25 OHD se consideran deficientes, y en los estudios analizados se establecen distintos puntos de corte para considerar el déficit. La mayoría utilizó para definir hipovitaminosis $\mathrm{D}$, niveles inferiores a $10 \mathrm{ng} / \mathrm{dl}$, valores que son considerados como déficit severo por la mayoría de las sociedades científicas debido a que el riesgo de raquitismo y osteomalacia es alto por debajo de este umbral, incluso con niveles adecuados de calcio. Este hallazgo puede llevar a subestimar aún más el déficit de vitamina $\mathrm{D}^{(16-19)}$.

La Sociedad de Endocrinología de Estados Unidos, la Academia Americana de Pediatría (AAP) y la Sociedad Italiana de Pediatría establecen déficit de vitamina $\mathrm{D}$ a valores por debajo de $20 \mathrm{ng} / \mathrm{ml}$, déficit severo niveles inferiores a $10 \mathrm{ng} / \mathrm{ml}$ e insuficiencia a valores entre 21-29 ng/ml ${ }^{(12,19,20)}$. En el año 2011, y tras la revisión de la literatura científica disponible, el Instituto de Medicina (IOM) concluyó que niveles de 25 OHD de $20 \mathrm{ng} / \mathrm{ml}$ cubren los requerimientos de $97,5 \%$ de la población general, y ese sería el valor de referencia. Si bien los niveles de $30 \mathrm{ng} / \mathrm{ml}$ cubre el $100 \%$ de la población, por lo cual valores menores a $30 \mathrm{ng} / \mathrm{ml}$ se consideran como insuficiencia por la mayoría de las sociedades científicas $^{(19-22)}$.

En uno de los artículos, la media de $25 \mathrm{OHD}$ antes de comenzar el estudio era de $14,45 \mathrm{ng} / \mathrm{dl}$, por lo cual existe un déficit previo al comienzo del análisis. En otro de los estudios, si bien se encontró solamente $13 \%$ de déficit de vitamina $\mathrm{D}(<10 \mathrm{ng} / \mathrm{dl})$, todos los pacientes incluidos presentaban niveles inferiores a $30 \mathrm{ng} / \mathrm{dl}$, valores de insuficiencia según la Sociedad de Endocrinología de Estados Unidos ${ }^{(15,17)}$.

El único estudio que analizó la variabilidad estacional de los niveles de vitamina $\mathrm{D}$ evidenció una tendencia decreciente en dichos niveles previo al inicio del tratamiento y pasados los tres años. Dicho descenso fue más pronunciado con el uso de AE. Esto indicaría que la exposición solar y la estación del año influye en los niveles de vitamina $\mathrm{D}$ y con terapia $\mathrm{AE}$ se reduce aún más ${ }^{(18)}$.

El estudio retrospectivo que incluye el mayor número de pacientes $(\mathrm{n}=596)$ y una prevalencia de las más altas halladas, evidencia además una diferencia de mayor riesgo de hipovitaminosis con los AE inductores. Como se mencionó con anterioridad, esto se debería a que dichos $\mathrm{AE}$ estimulan el metabolismo de la vitamina $\mathrm{D}$, ocasionando un descenso en sus niveles plasmáticos ${ }^{(16)}$.

Otro aspecto destacable de los estudios analizados fueron los criterios estrictos de exclusión, dejando fuera aquellas situaciones clínicas que podían afectar los valores de vitamina $\mathrm{D}$, como insuficiencia renal, enfermeda- 
des malabsortivas, medicamentos que alteren su metabolismo, entre otros.

Considerando lo analizado puede decirse que la relación teórica entre el uso de $\mathrm{AE}$ y déficit de vitamina $\mathrm{D}$ se corrobora en estos estudios clínicos, tanto en pacientes adultos como pediátricos. Además, es probable que este efecto no se restrinja a los AE inductores (asociación ya conocida), sino también a los AE no inductores de las enzimas hepáticas, incluso los inhibidores de éstas. Esto podría deberse a que dicho $\mathrm{AE}$ inhibe la enzima 25 hidroxilasa, la cual participa en la bioactivación de la vitamina D.

Con los escasos estudios disponibles, el número de pacientes incluidos y la no representatividad de todos los $\mathrm{AE}$, no es posible analizar completamente si hay diferencias potenciales entre ellos.

Es necesario continuar caracterizando esta asociación, en cuanto a su magnitud, factores de riesgo, severidad, evolución y modificación tras la suplementación.

Las recomendaciones a nivel nacional e internacional acerca de la suplementación profiláctica de vitamina $\mathrm{D}$ en pacientes que reciben medicación antiepiléptica está documentada. Para la población adulta, la Sociedad de Endocrinología de Estados Unidos y el IOM sugiere que los adultos que consumen AE, glucocorticoides, antifúngicos y antirretrovirales deben recibir dos a tres veces más vitamina $\mathrm{D}$ para satisfacer los requerimientos corporales. La dosis diaria recomendada es de 600 UI/día ${ }^{(12)}$. La AAP y la Sociedad Italiana de Pediatría establecen que los requerimientos basales de vitamina $\mathrm{D}$ son de 400 UI en menores de 1 año y de 600 UI a 1.000 UI en mayores de 1 año hasta los 18 años ${ }^{(19,20)}$. La Sociedad Uruguaya de Pediatría recomienda la administración de vitamina $\mathrm{D}$ independientemente de la edad, si el niño recibe $\mathrm{AE}$, tiene obesidad mórbida o afecciones cutáneas que impiden tomar sol, o no se expone al sol por diversas razones. Las dosis profilácticas de vitamina $\mathrm{D}$ recomendadas es de $400 \mathrm{IU} /$ día. La pauta de tratamiento en caso de déficit documentado es mayor ${ }^{(13)}$.

En cuanto a las recomendaciones acerca del tamizaje de vitamina $\mathrm{D}$ en la población que recibe $\mathrm{AE}$, la AAP determina que la evidencia es insuficiente para recomendar screening universal. Por otro lado, la Sociedad de Endocrinología de Estados Unidos recomienda realizarlo en individuos con riesgo, esto es, niños obesos, con síndromes malabsortivos, individuos hispanos y de raza negra, y que se encuentren en tratamiento con AE, corticoides, antifúngicos y medicación antirretroviral.

En base a la evidencia actual, si la dosificación de vitamina D se encuentra disponible (si es accesible y asequible) es razonable realizar su determinación en pacientes con factores de riesgo de hipovitaminosis, como es el caso de pacientes que reciben AE. De no estar disponible la dosificación, o de no ser costo-efectiva su realización, la profilaxis con vitamina $\mathrm{D}$ parece ser racional. En caso de déficit, es importante realizar tratamiento y monitorización.

Se deben considerar las implicancias clínicas del déficit de vitamina D en la población, ya que si bien se ha relacionado su déficit a alteraciones en la densidad ósea y enfermedades extra-óseas, aún existe controversia sobre el impacto real de su suplementación en variables duras como, por ejemplo, riesgo de fracturas, dado que su presencia se relaciona también con otros factores $^{(23-26)}$.

En Uruguay no existen datos acerca de los niveles séricos de vitamina $\mathrm{D}$ en pacientes tratados con $\mathrm{AE}$ y existen escasos estudios que evalúen niveles de vitamina $\mathrm{D}$ en la población. Se destaca la importancia de continuar realizando estudios en nuestro país para conocer la epidemiología local y poder establecer recomendaciones acordes para pacientes que utilizan $\mathrm{AE}$ y otros con otros factores de riesgo.

Actualmente se encuentra en desarrollo un estudio a cargo de docentes del Departamento de Farmacología y Terapéutica de la Facultad de Medicina de la Universidad de la República, que busca conocer la prevalencia del déficit de vitamina $D$ en pacientes que reciben AE y en la población general, incluyendo adultos y niños.

\section{Abstract}

Vitamin D regulates skeletal and extraskeletal functions. Hypovitaminosis D is a health problem that is now emerging again. This condition may be determined by several factors (sun exposure, bad absortion, obesity, sedentary life and exposure to antiepileptics). The study aimed to review evidence on the association between hipovitaminosis D and treatment with antiepileptics.

Method: we conducted a descriptive review of the evidence available in Pubmed and Scielo databases in the last 10 years.

Results: we analysed 5 studies that met the search criteria we had previously set. Four of the studies were prospective and 2 had been carried out in India (one of them in children exposed to carbamazepine and the other one in adults with diphenylhydantoin or valproic acid), another one in Greece in children with carbamazepine or valproic acid exposure, and the deficit was in all three cases; the fourth study was conducted in Germany in children exposed to several antiepileptics and no association was found. A restrospective study was carried out in United States in adults exposed to several antiepileptics and an association was found in this case.

Discussion: we point out the scarce number of studies found, they are observational, inlcude few patients 
and are short. It was evident that hipovitaminosis D was rather frequent although a causal relationship cannot be established. The extent of the problem is still not clear as there is no agreement on the Vitamin D levels that are to be regarded as defficient. Vitamin D prophylaxis in antiepileptic patients seems to be reasonable in spite of the limited information available. The association and its clinical implications need to be further charactertized.

\section{Resumo}

Objetivo: a vitamina D regula funções ósseas e extra ósseas. A hipovitaminose D está reemergindo como problema de saúde. Pode ser causada por diversos fatores (exposição solar, má absorção, obesidade, sedentarismo e exposição a antiepilépticos). O objetivo deste trabalho foi revisar a evidencia sobre a associação entre a hipovitaminose $\mathrm{D}$ e o tratamento com antiepilépticos.

Métodos: realizou-se uma revisão descritiva da evidencia disponível nos últimos 10 anos nas bases de dados Pubmed e Scielo.

Resultados: cinco estudos que cumpriam com os critérios de inclusão foram analisados. Quatro eram prospectivos: 2 realizados na Índia -um em crianças expostas a carbamazepina e outro em adultos com difenil-hidantoína ou ácido valpróico-, outro na Grécia em crianças tratadas com carbamazepina ou ácido valpróico; em todos evidenciou-se o déficit; o quarto estudo foi realizado na Alemanha em crianças expostas a vários antiepilépticos e não se encontrou associação. Um estudo retrospectivo, realizado nos Estados Unidos em adultos expostos a vários antiepilépticos não encontrou associação.

Discussão: destaca-se o número reduzido de estudos encontrados, sendo a maioria observacionais, com poucos pacientes e de curta duração. Evidencia-se que a hipovitaminose $\mathrm{D}$ foi frequente embora o tipo de estudo não permita estabelecer uma relação causal. A magnitude do problema ainda não é clara pois não existe consenso sobre que níveis de vitamina $\mathrm{D}$ são considerados deficientes. A profilaxia com vitamina $\mathrm{D}$ em pacientes com antiepilépticos parece racional apesar das limitações da informação disponível. É necessário continuar caracterizando a associação e suas implicações clínicas.

\section{Bibliografía}

1. Vitamina D: evidencias y controversias. Boletín INFAC. 2012;20(2):1-6. Disponible en: https://www.osakidetza.euskadi.eus/contenidos/informacion/cevime_infac/eu_miez/adjuntos/INFAC_Vol_20_n_2.pdf [Consulta: 2 de octubre de 2018].

2. Bouillon R. Vitamin D and extraskeletal health. UpToDate. 2017:1-22.
3. Mithal DA, Wahl J, Bonjour P, Burckhardt P, Dawson-Hughes B, Eisman JA, et al. Global vitamin D status and determinants of hypovitaminosis D. Osteoporos Int 2009;(20):1807-1820

4. Beerhost K, Van der Krujis S, Verschuure P, Aldenkamp A. Bone disease during chronic antiepileptic drug therapy: General versus specific risk factors. Journal of the Neurological Sciences. 2013;331:20-24.

5. Ganji V, Hang X, Tangpricha V. Serum 25-hydroxivitamin D concentrations and prevalence estimates of hypovitaminosis D in the U.S population based on assay-adjusted data. J. Nutr. 2012;142(3):498-507.

6. Puche R. Sobre la prevalencia de hipovitaminosis D en Argentina. Medicina 2015;75(3):183-186.

7. Solá L, Leyún N, Díaz JC, González S, Sehabiague C, Parodi $\mathrm{K}$, Olascoaga $\mathrm{A}$, et al. Asociación de déficit de vitamina D y anemia en pacientes en hemodiálisis crónica. Rev Méd Urug 2014; 30(3):157-163.

8. Gómez F, Bottaro S, Cóppola F , Tomasso G, Rocha V, Giménez C. Prevalencia del déficit de vitamina D en una población de embarazadas que se asisten en el Centro Hospitalario Pereira Rossell. Rev Med Urug 2016; 32(2):87-97.

9. Mendoza B, Ronco A. Estacionalidad de la vitamina D plasmática: su importancia en la clínica y en la salud. Rev Méd Urug 2016; 32(2):80-86.

10. Tomita S, Ohnishi J, Nakano M, et al. The effect of anticonvulsant drugs on vitamin D3-activating cytochrome P-450-linked monooxygenase systems. J Steroid Biochem Mol. 1991;39:479-485.

11. American Academy of Pediatrics. Clinical report. Optimizing Bone Health in Children and Adolescents. PEDIATRICS. 2014;134(4):1229-1243.

12. Holick M, Binkley N, Bischoff-Ferrari H, Gordon C, Hanley D, Heaney R, et al. Evaluation, Treatment, and Prevention of Vitamin D Deficiency: an Endocrine Society Clinical Practice Guideline. J Clin Endocrinol Metab.2011;96(7):1911-1930.

13. Estefanell C, Olivera R, Satriano R, Tanzi MN, Donangelo C, Salmenton M, Giachetto G, et al. Pauta de vitamina D. Arch Pediatr Urug. 2012;83(1):31-34.

14. Krishnamoorty G, Nair R, Sundar U, Kini P, Shrivastava M. Early Predisposition to osteomalacia in Indian adults on phenytoin or valproate monotherapy and effective prophylaxis by simultaneous supplementation with calcium and 25-hydroxy vitamin D at recommended daily allowance dosage: A prospective study. Neurology India. 2010;58: 213-217.

15. Misra A, Aggarwal A, Singh O, Sharma S. Effect of carbamazepine therapy on vitamin D and parathormone in epileptic children. Pediatr Neurol 2010;43(5):320-324.

16. Teagarden D, Meador K, Loring D. Low Vitamin D levels are common in patients with epilepsy. Elsevier. 2014;108:1352-1356.

17. Borusiak $\mathbf{P}$, Langer T, Heruth $\mathbf{M}$, Karenfort M, Bettendorf U, Jenke A. Antiepileptics drugs and bone metabolism 
in children: data from 128 patients. J Child Neurol. 2012;00(0):1-8.

18. Nicolaidou P, Georgouli H, Kotsalis H, Matsinos Y, Papadopoulou A, Fretzayas A. Effects of Anticonvulsant Therapy on Vitamin D Status in Children: Prospective Monitoring Study Journal of Child Neurology. 2006; 21(3):205-209.

19. Saggese G, Vierucci F , Prodam F, Cardinale F, Cetin I, Chiappini E et al. Vitamin D in pediatric age: consensus of the Italian Pediatric Society and the Italian Society of Preventive and Social Pediatrics, jointly with the Italian Federation of Pediatricians. Italian Journal of Pediatrics (2018) 44:51

20. Wagner C, Greer F. Prevention of Rickets and Vitamin D Deficiency in Infants, Children, and Adolescents. PEDIATRICS. 2008;122(5):1142-1152.

21. Institute of Medicine. Dietary reference intakes for calcium and vitamin D. 2011. Washington, DC: The National Academies Press)
22. Ross C, Manson E, Abrams S. The 2011 Report on Dietary Reference Intakes for Calcium and Vitamin D from the Institute of Medicine: What Clinicians Need to Know. J Clin Endocrinol Metab. 2011; 96(1):53-58.

23. García Vadillo J. Suplementos de calcio y vitamina D ¿para todos?: Pros. Reumatol Clin. 2011;7(S2):S34-S39

24. Loza Santamaría E. Suplementos de calcio y vitamina D ¿para todos?: Contras. Reumatol Clin. 2011;7(S2):S40-S45

25. García-Franco A, Navarro D, Corrochano E. Vitamina D: el traje nuevo del Rey Sol. Aten Primaria. 2019;51(2):57-58

26. Mengual Gil JM. Vitamina D por encima del año de vida, ¿es necesaria o está de moda? En: AEPap (ed.). Curso de Actualización Pediatría 2018. Madrid: Lúa Ediciones 3.0; 2018. p. 17-30.

\section{Contribución de autores}

Todos los autores participaron en igual medida en todas las etapas de elaboración y proceso del trabajo.

Valentina Catenaccio, https://orcid.org/0000-0003-4236-610X

Agustina De Santis, https://orcid.org/0000-0002-6630-0925

Noelia Speranza, https://orcid.org/0000-0002-3330-9974 